\title{
Niños y adolescentes expuestos ambientalmente a mercurio, en diferentes municipios de Colombia
}

\author{
Children and adolescents exposed environmentally to \\ mercury in different municipalities in Colombia
}

\author{
Marien Palma-Parra ${ }^{1}$; Ma-Nathalia Muñoz-Guerrero ${ }^{1}$; Oscar Pacheco-Garcia ${ }^{1}$; Yamileth Ortiz-Gomez ${ }^{1}$; Sonia-M Díaz-C ${ }^{1}$
}

Forma de citar: Palma-Parra M, Muñoz-Guerrero MN, Pacheco-García O, Ortiz-Gomez Y, Díaz S. Niños y adolescentes expuestos ambientalmente a mercurio en diferentes municipios de Colombia. Rev Univ Ind Santander Salud. 2019; 51(1): 43-52. doi: http://dx.doi.org/10.18273/revsal.v51n1-2019005 @c (1)

\section{Resumen}

Objetivo: Establecer la exposición ambiental a mercurio, para identificar el impacto sobre la salud de la población de niños y adolescentes en cuatro departamentos de Colombia. Metodología: Se llevó a cabo un estudio descriptivo transversal entre 2014 - 2015, en once municipios de los departamentos de Bolívar, Sucre, Antioquia y Córdoba y dentro de este se realizó un estudio anidado con un grupo de 63 niños y uno de 37 adolescentes de estos municipios. Resultados: El promedio de tiempo de residencia estuvo entre 6 y 9 años y se encontró alta frecuencia de consumo de pescado. El 6,3\% de los niños y el 29,7\% de los adolescentes, excedieron el límite permisible de mercurio en sangre y el $50,8 \%$ de los niños y el $46,0 \%$ de los adolescentes excedieron el límite permisible en cabello. En cuanto a los síntomas referenciados por los participantes, hubo diferencia estadísticamente significativa $(\mathrm{p}>0,05)$, entre la población de niños y la de adolescentes en salivación excesiva (15,9\%), caída del cabello (18,7\%), insomnio $(29,0 \%)$, nerviosismo $(19,6 \%)$, debilidad $(18,0 \%)$ y pérdida de memoria $(17,3 \%)$. Sabor metálico en la boca $(8,4 \%)$, pérdida de peso $(23,4 \%)$, temblor $(21,5 \%)$, problemas de concentración $(17,9 \%)$ y dolor torácico $(17,9 \%)$ no mostraron diferencia entre estos dos grupos. Conclusión: Los niños y adolescentes que residen en áreas de explotación de oro o influenciados por la minería de oro, se encuentran expuestos a mercurio, lo que implica un riesgo elevado para su salud.

Palabras clave: Niños; Adolescentes; Exposición ambiental; Mercurio; Muestras biológicas.

\begin{abstract}
Objective: It was proposed to establish the environmental exposure to mercury, to identify the impact on the health of the population of children and adolescents in four departments of Colombia. Methodology: A cross-sectional descriptive study was carried out between 2014 - 2015, in eleven municipalities of the departments of Bolívar, Sucre, Antioquia and Córdoba and within this a nested study was carried out with a group of 63 children and 37 adolescents from these municipalities. Results: Average residence time between 6 and 9 years and a high frquency of fish consumption was found. $6.3 \%$ of children and $29.7 \%$ of adolescents exceeded the allowable limit of

1. Instituto Nacional de Salud. Bogotá, DC, Colombia.

Correspondencia: Sonia Mireya Díaz. Dirección: Calle 26 \# 51-20. Teléfono: +57 12207700 ext 1216. Correo electrónico: sdiaz@ins.gov.co


mercury in blood and $50.8 \%$ of children and $46.0 \%$ of adolescents exceeded the permissible limit on hair. Regarding the symptoms referenced by the participants, there was a statistically significant difference $(p>0.05)$ between the population of children and that of adolescents in excessive salivation (15.9\%), hair loss (18.7\%), insomnia (29.0\%), nervousness $(19.6 \%)$, weakness $(18.0 \%)$ and memory loss $(17.3 \%)$. Metallic taste in the mouth $(8.4 \%)$, weight loss $(23.4 \%)$, tremor $(21.5 \%)$, concentration problems $(17.9 \%)$ and chest pain $(17.9 \%)$ did not show difference between the two groups. Conclusions: Children and adolescents who reside in areas of gold exploitation or who are influenced by gold mining are exposed to mercury, which implies a high risk to their health.

Keywords: Children; Adolescents; Environmental exposure; Mercury; Biological samples.

\section{Introducción}

El mercurio es un contaminante mundial, que llega al medio ambiente por actividades humanas y en pequeña proporción desde la corteza terrestre ${ }^{1}$. Desde una perspectiva global, las fuentes más importantes de mercurio son las asociadas a actividades humanas, como la minería de oro, la cual es responsable del $37 \%$ de todo el mercurio antropogénico ${ }^{2}$, el uso de combustibles fósiles y los vertimientos industriales. Gran parte de los vapores de mercurio llegan a la atmósfera, o este se deposita directamente a los cuerpos de agua cercanos sin ningún tipo de tratamiento y es transformado por las bacterias en metilmercurio, biomagnificándose a través de la cadena trófica y llegando a los peces y a quienes los consumen ${ }^{3-9}$. Una vez absorbido, el $\mathrm{Hg}$ se une a los eritrocitos ${ }^{10} \mathrm{y}$ a las proteínas plasmáticas, depositándose en el sistema nervioso ${ }^{11}$, en los riñones y en el hígado y otros órganos ${ }^{12}$.

La región de la Mojana, Figura 1, de la que hacen parte algunos municipios de nuestro estudio, es una subregión ubicada en la parte norte de Colombia, caracterizada por ser una zona de humedales productivos, perteneciente a la Depresión Momposina, con la función ambiental de regular los cauces de los ríos Magdalena, Cauca y San Jorge. Ha estado impactada desde hace mucho tiempo por la minería de oro que se lleva a cabo en algunos de estos municipios o en zonas aledañas, la cual puede ser de aluvión o de filón. La mayor actividad minera se encuentra en la llanura del río Magdalena, parte baja de los ríos Cauca y San Jorge, norte de Antioquia y parte sur de Córdoba, con uso profuso de mercurio y cianuro ${ }^{13}$.

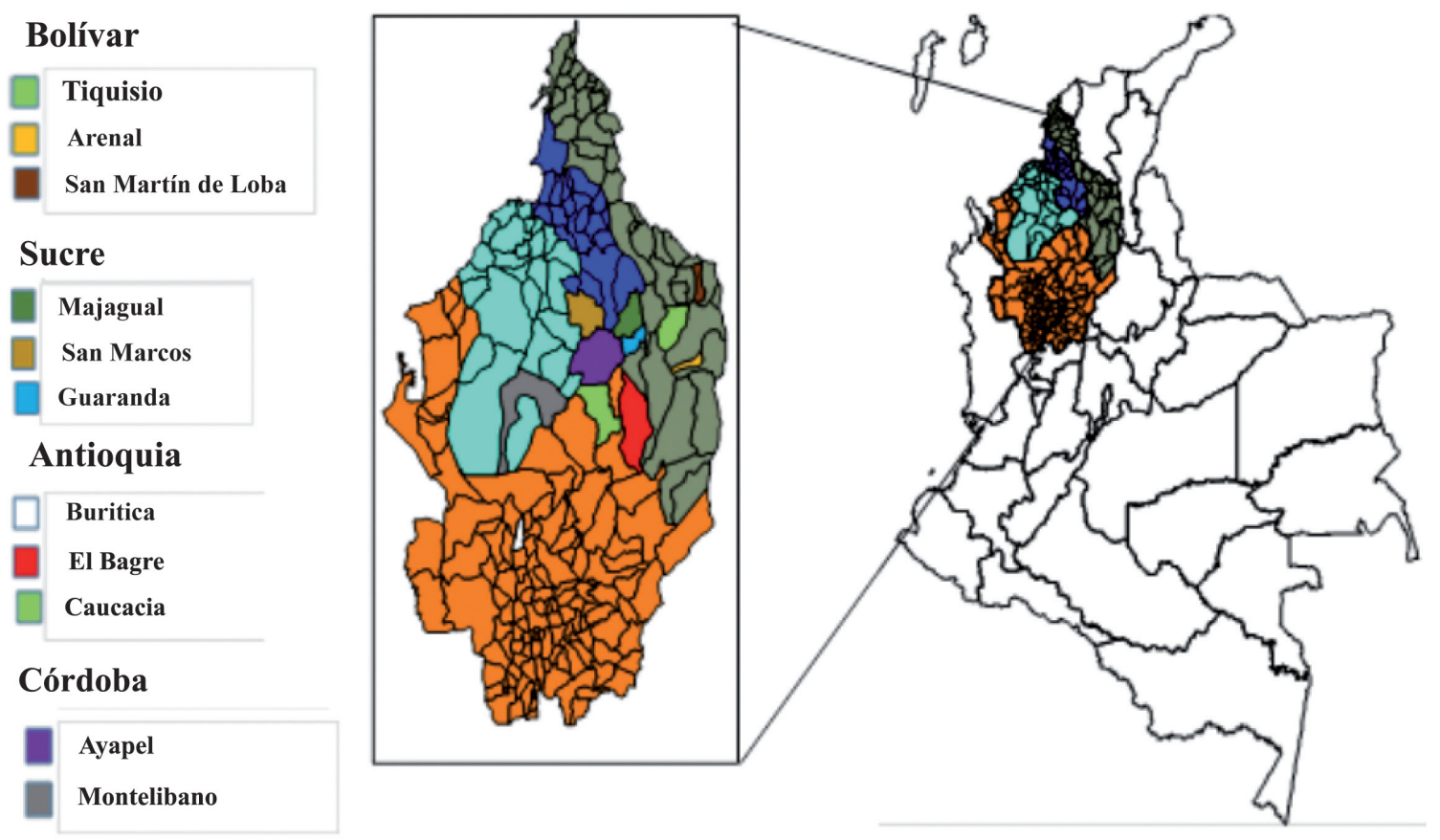

Figura 1. Mapa de la región de estudio 
Los niños son más sensibles a ciertas exposiciones ambientales $^{14-18}$, por tener frecuencia respiratoria más rápida, superficie pulmonar más grande ${ }^{19,20}$, tener una estatura menor que los adultos y realizar actividades como gatear o jugar en el suelo, donde las concentraciones de vapor de mercurio son más altos $^{21-23}$. La contaminación del aire en espacios cerrados y exteriores, la insalubridad del agua, la falta de saneamiento y la higiene inadecuada, causan la muerte de 1,7 millones de niños menores de cinco años. A estas exposiciones se atribuye la tercera parte de las muertes en el grupo de edad de $0-19$ años ${ }^{24}$, más de una cuarta parte de las defunciones de niños menores de cinco años ${ }^{25}$.

El mercurio produce estrés oxidativo en el sistema nervioso central y la conducción neura ${ }^{26}$ ocasionando una extrema afectación a nivel neurológico y neurocomportamental en los niños, déficit en las capacidades sensoriales, $\operatorname{cognitivas}^{27}$ y psicológicas, lo que se refleja en puntajes y habilidades de memoria visoespacial y verbales más bajos en la escuela ${ }^{21,28}$, inadecuado desarrollo de las funciones que intervienen en los procesos de aprendizaje y desempeño escolar, con afectaciones en su personalidad y en relaciones sociales ${ }^{21,29-31}$. Los efectos tóxicos del mercurio, dependen de la exposición, en tiempo, cantidad, frecuencias, aunque se ha descrito una marcada variabilidad de la respuesta personal al mercurio, entre los diferentes grupos de población ${ }^{32}$.

En el cabello, el mercurio se integra reflejando la historia de la exposición pasada en periodos largos, porque puede permanecer en el cabello por más de 11 años sin cambio ${ }^{12}$, lo que permite que esta muestra sea la de elección para mediciones de exposición crónica. Una de las fuentes más frecuentes de esta exposición es el consumo de pescado. En algunos estudios realizados en niños de diferentes edades, se ha encontrado que la frecuencia de consumo de pescado se relacionó directamente con las concentraciones de mercurio en cabello ${ }^{33-36}$. Igualmente, la exposición de madres a mercurio por consumo de pescado ha mostrado que incrementos de $1 \mathrm{mg}$ por encima de $10 \mathrm{mg} / \mathrm{g}$ de mercurio en cabello en la madre, se asocia con una disminución del coeficiente intelectual de los niños, alteraciones en el neurodesarrollo, en el lenguaje, la memoria y funciones motoras y adicionalmente, bajo peso al nacer8, ${ }^{87-39}$, y daño genotoxico aun con bajas concentraciones de exposición a mercurio, por lo que se usa como marcador temprano de daño a nivel genético ${ }^{40,41}$.
En Colombia, no se han realizado muchos estudios que aborden la complejidad del problema de exposición a mercurio en población de niños y adolescentes, a pesar de que se conoce el efecto toxico de este contamínate y el daño en la salud de la población. Se conoce el realizado por Vargas y Quiroz en escolares en el municipio de Segovia Antioquia, con el fin de establecer la prevalencia de alteraciones neuropsicológicas en lenguaje, memoria, funciones ejecutivas y atención de los escolares de ese municipio ${ }^{41}$.

En este artículo se describen los resultados de un estudio anidado dentro de un trabajo de investigación en cuatro departamentos del norte de Colombia, en el que se cuantifica la exposición a mercurio en la población de niños y adolescentes de esta zona, y evidencia la magnitud del problema en estos dos grupos poblacionales.

\section{Metodología}

Se llevó a cabo un estudio descriptivo transversal durante el 2014 - 2015, en once municipios de Colombia: Arenal, Tiquisio y San Martin de Loba, Majagual, Guaranda, San Marcos, Caucasia, El Bagre, Buriticá, Ayapel y Montelibano, y dentro de este se realizó un estudio anidado con un grupo de niños y un grupo de adolescentes de dichos municipios. Los participantes se seleccionaron por conveniencia, como criterio de inclusión se tuvo en cuenta que estuvieran viviendo al menos seis meses en la zona y reportaran consumir pescado del lugar; la aplicación de un muestreo aleatorio no fue posible debido a la dispersión de la población en la zona.

Se tomaron muestras de sangre y de cabello, como indicadores de exposición aguda y crónica, se realizó una entrevista a cada participante, acompañado de su representante. La información se recopiló por medio de un cuestionario, adaptado del instrumento de evaluación en salud del Global Mercury Project ${ }^{40}$, el cual incluyó variables sociales y demográficas, hábitos alimenticios, signos y síntomas relacionados con la exposición a mercurio. A cada individuo seleccionado se le tomó una muestra de $10 \mathrm{ml}$ de sangre por punción venosa en tubos con anticoagulante (EDTA), mantenidos en refrigeración hasta su análisis. Asimismo, se recolectaron aproximadamente $10 \mathrm{~g}$ de cabello conservado en bolsas de polietileno a temperatura ambiente para determinación de mercurio total. Las muestras fueron procesadas en el Instituto Nacional de Salud, y se analizaron en el equipo DMA-80 TriCell Milestone de acuerdo al método 
EPA 7473 (descomposición térmica, amalgamación y espectroscopia de absorción atómica) ${ }^{40}$. Los valores de referencia utilizados fueron $(5 \mu \mathrm{g} / \mathrm{L}$ en sangre total, $1 \mu \mathrm{g} / \mathrm{g}$ de cabello) reportados por $\mathrm{OMS}^{41}$. También se recolectaron muestras de pescado de las especies más consumidas en cada municipio, estas se procesaron por espectrofotometría de absorción atómica por vapor frio (CVAAS) (40). Los valores de referencia utilizados fueron de $0.5 \mathrm{mg} / \mathrm{kg}$ para especies no carnívoras y de $1 \mathrm{mg} / \mathrm{kg}$ para especies carnívoras ${ }^{42}$.

La información se sistematizó en una base de datos en el programa SPSS V.22. Se realizó análisis univariado con distribuciones de frecuencias, medidas de tendencia central y dispersión, se utilizó la t de Student, U de Mann-Whitney y chi cuadrado, para la comparación de las variables cuantitativas y categóricas y análisis bivariado calculando OR con sus respectivos intervalos de confianza. El nivel de significancia de $p$ fue de 0,05 .

\section{Resultados}

La muestra quedó conformada por 100 sujetos, 63 $(63,0 \%)$ niños con edades entre 2 y 10 años y 37 $(37,0 \%)$ adolescentes entre 11 y 17 años, con una media de edad de 9,4 años. La mayor frecuencia de exposición fue la ambiental, solo cuatro adolescentes informaron trabajar en minería.

Tabla 1. Características sociodemográficas de los participantes niños y adolescentes, Colombia, 2015.

\begin{tabular}{|c|c|c|c|c|c|c|c|c|}
\hline \multirow{2}{*}{ Variable } & \multirow{2}{*}{ Categorías } & \multicolumn{2}{|c|}{ Niños (0-10 años) } & \multicolumn{2}{|c|}{ Adolescentes (11-17 años) } & \multicolumn{2}{|c|}{ Total } & \multirow{2}{*}{$\mathbf{p}$} \\
\hline & & n (63) & $\%$ & n (37) & $\%$ & n (100) & $\%$ & \\
\hline \multirow{2}{*}{$\begin{array}{l}\text { Tipo de } \\
\text { exposición }\end{array}$} & Ocupacional & 0 & $0,0 \%$ & 4 & $4,0 \%$ & 4 & $4,0 \%$ & \multirow{2}{*}{0,008} \\
\hline & Ambiental & 63 & $63,0 \%$ & 33 & $33,0 \%$ & 96 & $96,0 \%$ & \\
\hline \multirow{2}{*}{ Sexo } & Masculino & 31 & $31,0 \%$ & 17 & $17,0 \%$ & 48 & $48,0 \%$ & \multirow{2}{*}{0,753} \\
\hline & Femenino & 32 & $32,0 \%$ & 20 & $20,0 \%$ & 52 & $52,0 \%$ & \\
\hline \multirow{4}{*}{$\begin{array}{l}\text { Sistema de } \\
\text { seguridad social } \\
\text { en salud }\end{array}$} & Contributivo & 12 & $12,0 \%$ & 5 & $5,0 \%$ & 17 & $17,0 \%$ & \multirow{4}{*}{0,259} \\
\hline & Subsidiado & 49 & $49,0 \%$ & 28 & $28,0 \%$ & 77 & $77,0 \%$ & \\
\hline & No afiliado & 2 & $2,0 \%$ & 4 & $4,0 \%$ & 6 & $6,0 \%$ & \\
\hline & Analfabeto & 6 & $6,0 \%$ & 1 & $1,0 \%$ & 7 & $7,0 \%$ & \\
\hline \multirow[t]{9}{*}{ Educación } & Primaria incompleta & 51 & $51,0 \%$ & 15 & $15,0 \%$ & 66 & $66,0 \%$ & \multirow[t]{2}{*}{0,000} \\
\hline & Secundaria incompleta & 2 & $2,0 \%$ & 21 & $21,0 \%$ & 23 & $23,0 \%$ & \\
\hline & Bolívar & 17 & $17,0 \%$ & 6 & $6,0 \%$ & 23 & $23,0 \%$ & \\
\hline & Tiquisio & 7 & $7,0 \%$ & 0 & $0,0 \%$ & 7 & $7,0 \%$ & \\
\hline & San Martin de Loba & 6 & $6,0 \%$ & 3 & $3,0 \%$ & 9 & $9,0 \%$ & \\
\hline & Arenal & 4 & $4,0 \%$ & 3 & $3,0 \%$ & 7 & $7,0 \%$ & \\
\hline & Sucre & 14 & $14,0 \%$ & 11 & $11,0 \%$ & 25 & $25,0 \%$ & \\
\hline & Guaranda & 6 & $6,0 \%$ & 3 & $3,0 \%$ & 9 & $9,0 \%$ & \\
\hline & Majagual & 0 & $0,0 \%$ & 1 & $1,0 \%$ & 1 & $1,0 \%$ & \\
\hline \multirow[t]{8}{*}{ Localización } & San Marcos & 8 & $8,0 \%$ & 7 & $7,0 \%$ & 15 & $15,0 \%$ & \multirow[t]{8}{*}{0,296} \\
\hline & Antioquía & 18 & $18,0 \%$ & 10 & $10,0 \%$ & 28 & $28,0 \%$ & \\
\hline & Buriticá & 6 & $6,0 \%$ & 0 & $0,0 \%$ & 6 & $6,0 \%$ & \\
\hline & Caucasia & 9 & $9,0 \%$ & 7 & $7,0 \%$ & 16 & $16,0 \%$ & \\
\hline & El Bagre & 3 & $3,0 \%$ & 3 & $3,0 \%$ & 6 & $6,0 \%$ & \\
\hline & Córdoba & 14 & $14,0 \%$ & 10 & $10,0 \%$ & 24 & $24,0 \%$ & \\
\hline & Ayapel & 6 & $6,0 \%$ & 6 & $6,0 \%$ & 12 & $12,0 \%$ & \\
\hline & Montelibano & 8 & $8,0 \%$ & 4 & $4,0 \%$ & 12 & $12,0 \%$ & \\
\hline
\end{tabular}

La media de residencia en el lugar, en los niños fue de 6,1 años $\pm 2,9$ y en adolescentes de 9,6 años $\pm 4,5$. Con relación al consumo de pescado, se realizó un análisis proximal basado en la información familiar al respecto y se encontró que el 37,0\% (37) consumían pescado una vez al mes, el 30,0\% (30) dos a cuatro veces por semana, 
el 22,0\% (22) una vez por semana y el 6,0\% (6) a diario. De las 18 especies de pescado muestreadas, las de mayor consumo fueron en su orden mojarra, bagre y tilapia, las cuales presentaron un promedio de concentraciones de mercurio de $0,70 \mathrm{mg} / \mathrm{kg}, 0,87 \mathrm{mg} / \mathrm{kg}$ y $0,03 \mathrm{mg} / \mathrm{kg}$ respectivamente. La especie moncholo presentó 1,11 $\mathrm{mg} / \mathrm{kg}$ de mercurio, superando el límite permisible para las especies carnívoras. El 100\% de los encuestados reportó consumir agua del río cercano, quebrada o pozo.
En cuanto a la media de concentraciones de mercurio en muestras biológicas, se encontró que para sangre, el municipio de Montelibano presento el mayor promedio con un valor de $22,5 \mathrm{ug} / \mathrm{mL}$, seguido de Guaranda con 7,8 ug/mL y Buriticá con 5,1 ug/mL. Respecto de cabello, el municipio de Montelibano fue el de más alto valor con 3,25 ug/g, seguido de San Marcos con 3,17 ug/g y Buriticá con 2,3 ug/g.

Tabla 2. Concentraciones de mercurio en sangre y cabello por municipio de estudio, Colombia 2015.

\begin{tabular}{|c|c|c|c|c|c|c|c|c|c|}
\hline \multirow{2}{*}{ Municipio } & \multirow{2}{*}{$\mathbf{n}$} & \multicolumn{4}{|c|}{ Sangre ug/mL } & \multicolumn{4}{|c|}{ Cabello ug/g } \\
\hline & & Media & IC $95 \%$ & Mínimo & Máximo & Media & IC $95 \%$ & Mínimo & Máximo \\
\hline Tiquisio & 7 & - & - & - & - & 0,71 & $0,09-1,33$ & 0,29 & 2,22 \\
\hline $\begin{array}{l}\text { San Martin de } \\
\text { Loba }\end{array}$ & 9 & 1,8 & $0,52-3,07$ & 0 & 3,9 & 0,63 & $0,34-0,93$ & 0,19 & 1,27 \\
\hline Arenal & 7 & 2,04 & $-1,45-5,53$ & 0,3 & 6,9 & 1,77 & $0,46-3,08$ & 0,61 & 3,08 \\
\hline Guaranda & 9 & 7,83 & $-1,44-17,1$ & 1,04 & 23,12 & 1,63 & $0,18-3,08$ & 0,33 & 5,92 \\
\hline Majagual & 1 & - & - & - & - & - & - & - & - \\
\hline San Marcos & 15 & 2,65 & $-14,63-19,93$ & 1,29 & 4,01 & 3,17 & $2,04-4,29$ & 0,66 & 6,78 \\
\hline Buriticá & 6 & 5,11 & $2,03-8,19$ & 2,99 & 7,63 & 2,33 & $-1,19-5,85$ & 0,23 & 7,18 \\
\hline Caucasia & 16 & 2,32 & $0,59-4,04$ & 1,19 & 4,68 & 0,86 & $0,65-1,60$ & 0,38 & 1,59 \\
\hline El Bagre & 6 & 3,16 & $-12,52-18,85$ & 1,93 & 4,4 & 1,6 & $0,05-3,14$ & 0,55 & 4,44 \\
\hline Ayapel & 12 & 5,06 & $0,80-9,32$ & 1,33 & 13,7 & 1,88 & $0,99-2,76$ & 0,67 & 5,61 \\
\hline Montelíbano & 12 & 22,57 & $13,69-31,45$ & 17,4 & 30,1 & 3,25 & $1,18-5,31$ & 0,68 & 9,92 \\
\hline
\end{tabular}

Asimismo en la Figura 2, se observa que el 6,3\% (4) de los niños y el 29,7\% (11) de los adolescentes excedieron el límite permisible en sangre y el 50,8\% (32) de los

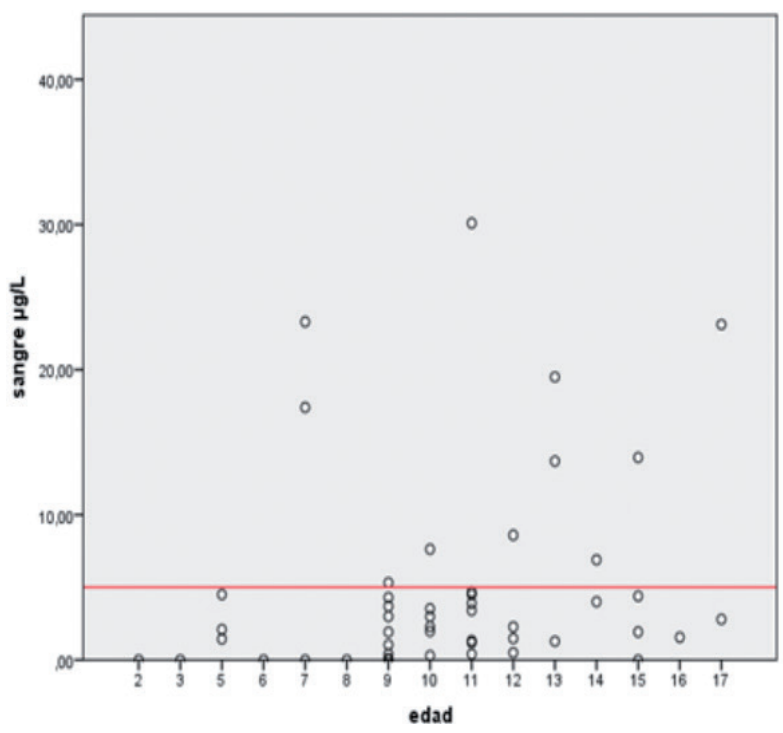

niños y el 46,0\% (17) de los adolescentes excedieron el límite permisible en cabello

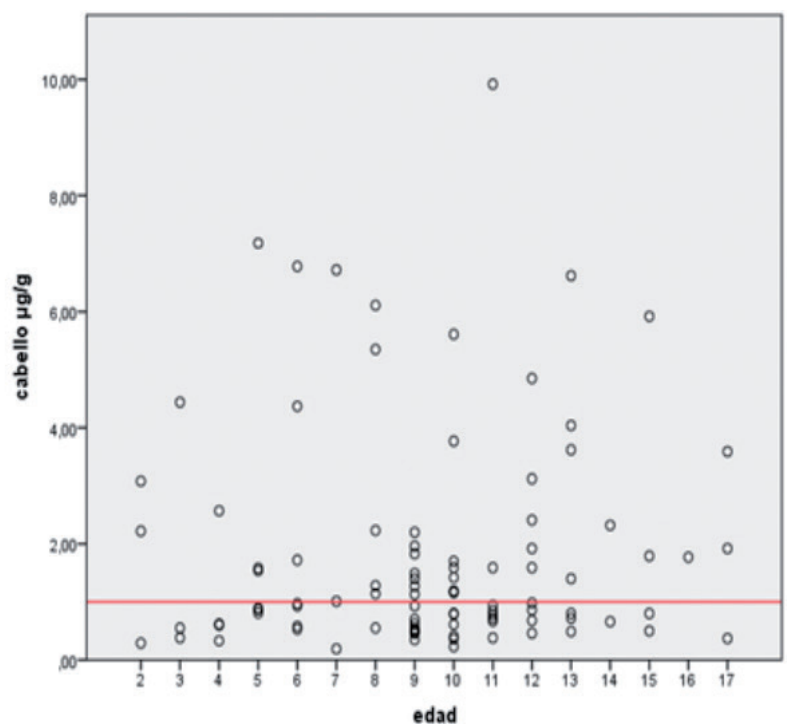

Figura 2. Concentraciones de mercurio en sangre y cabello de niños y adolescentes. Colombia, 2015. 
Las concentraciones promedio de mercurio en sangre no fueron estadísticamente significativos entre niños y adolescentes, según la prueba $U$ de Mann-Whitney $(\mathrm{p}=0.41)$; esto mismo sucedió con las concentraciones promedio de mercurio en cabello $(\mathrm{p}=0.94)$.

$\mathrm{Al}$ explorar la relación entre la frecuencia de consumo de pescado y las concentraciones de mercurio superiores a los valores permisibles en sangre y cabello, se halló que las frecuencias de consumo semanal y mensual fueron las de mayor reporte con 21 y 22 participantes respectivamente, con una relación estadísticamente significativa para cabello $(\mathrm{p}=0,010)$.

La razón entre los promedios de concentraciones de mercurio en sangre superiores al límite permisible y los promedios de las concentraciones inferiores al límite permisible fue 2.2 veces más en niños en comparación a los adolescentes, sin ser estadísticamente significativo (IC 95\% 0.54-8.63). Igualmente se evidenció una correlación lineal entre las concentraciones de mercurio en las dos matrices (coeficiente de Pearson $=$ $0,818$ y el valor de $p=0,000)$ (Figura 3).

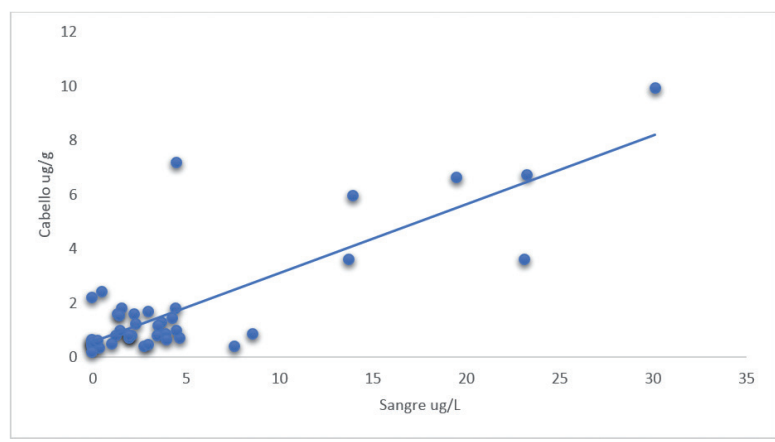

Figura 3. Correlación entre las concentraciones de mercurio en sangre y en cabello.

Durante la entrevista se indagó sobre síntomas asociados a intoxicación por mercurio y se encontró que la salivación excesiva $(15,9 \%)$, caída del cabello $(18,7 \%)$, insomnio $(29,0 \%)$, nerviosismo $(19,6 \%)$, debilidad $(18,0 \%)$ y pérdida de memoria $(17,3 \%)$ presentaron diferencias significativas $(\mathrm{p}<0,05)$ entre los dos grupos poblacionales frente a los niveles de mercurio superiores a los límites permisibles en cualquiera de las dos matrices, mientras que sabor metálico en la boca $(8,4 \%)$, pérdida de peso $(23,4 \%)$, temblor $(21,5 \%)$, problemas de concentración $(17,9 \%)$ y dolor torácico $(17,9 \%)$, no mostraron diferencia estadística.

\section{Discusión}

La contaminación ambiental por mercurio en Colombia, se da como consecuencia de emisiones actuales o pasadas, producto de la minería de oro. Infortunadamente, son pocas las medidas y controles que se han puesto en marcha para evitarlo y, en consecuencia, altas concentraciones de mercurio persisten en suelo, agua y sedimentos que se integran en la cadena alimentaria de los peces y de los consumidores de los mismos ${ }^{3,44}$.

La población de los municipios estudiados es afectada por la explotación minera que se realiza tanto en la zona, como en otros departamentos con los que comparten límites geográficos ${ }^{13}$. Es una población con difíciles condiciones de vida y alto porcentaje de necesidades básicas insatisfechas, que hacen que sus habitantes vean en la minería a pequeña escala, su fuente única de recursos, contaminando con mercurio su ambiente y el resto de habitantes de sus localidades.

Los niños y en general la población que reside en áreas de explotación de oro o cercanas a estas, se encuentran expuestos a contaminación por el mercurio que se utiliza en la extracción del metal, máxime cuando esta actividad se realiza en pequeña escala y de manera artesanal. Se encontraron niveles de mercurio por encima del límite permisible en la población de niños y adolescentes de los municipios incluidos en el estudio, por la exposición ambiental que resulta de consumo de pescado contaminado con metilmercurio y agua sin un tratamiento adecuado. Muchos adolescentes comienzan a trabajar con el mercurio desde temprana edad en las minas de explotación de oro $^{45}$, pero la mayoría, así no trabajen directamente con el metal, viven desde siempre en áreas cercanas a la extracción minera y por supuesto se contaminan ambientalmente. Es importante tener en cuenta además, el consumo de pescado, su fuente más cercana y casi única de proteína, situación que fue identificada en el análisis realizado, en el que la mayor frecuencia de consumo de pescado fue diaria, así como el autoreporte de consumo de agua del rio.

El conocimiento de los efectos que sobre la salud tienen los metales pesados como el mercurio, proviene en su mayoría de los estudios realizados en poblaciones con una exposición relativamente alta, como los trabajadores en la industria o en la minería de oro. 
Por ello, llama la atención los hallazgos sobre los síntomas reportados en niños y adolescentes, en especial el insomnio y nerviosismo, situación que siendo diferencial entre los grupos poblacionales podría estar relacionado de forma indirecta con la exposición ambiental, en nuestro caso se observó una media de tiempo de residencia de seis años en niños y nueve años en adolescentes, lo que puede significar que sus familias pueden llevar viviendo en el lugar mucho más tiempo y sus madres haber estado expuestas desde la gestación, a vapores de mercurio que en estos sitios de explotación de oro casi siempre están por encima de los límites que tiene establecidos la OMS para la exposición pública que es de $1.0 \mu \mathrm{g} / \mathrm{m}^{32}$. Esta situación se convierte en un factor de riesgo no solo para los trabajadores, sino también a las comunidades que se asientan cerca de los sitios de extracción y procesamiento del oro.

De la misma forma podría considerarse la exposición ocupacional, ya que el $15 \%$ de los participantes adolescentes informaron no haber terminado sus estudios primarios, característica común en esta zona, en donde existe deserción escolar para iniciar vida laboral en cualquier medio de trabajo, incluyendo la minería de oro.

De acuerdo con la OMS, en las poblaciones donde la exposición a $\mathrm{Hg}$ ocurre principalmente a través del consumo de pescado contaminado, la mayor parte del Hg total en la sangre y cabello es orgánico y puede usarse como una medida de la exposición a éste metal ${ }^{46}$. En las dos poblaciones de estudio, se encontraron concentraciones de mercurio en sangre y cabello por encima de los límites permisibles, marcándose más esta condición cuando se analizaron los datos de cabello, lo cual se relaciona de manera directa con la frecuencia de consumo de pescado informada por aproximadamente el $36 \%$ de los participantes, quienes consumían pescado con una frecuencia de entre diario y dos a cuatro veces por semana, tal como se describe en los hallazgos de un estudio realizado en niños en Malasia, en el cual se encontraron concentraciones de mercurio en cabello elevados asociados a la frecuencia de consumo de pescado ${ }^{47}$. Si se tiene en cuenta que entre los consumidores de pescado, el metilmercurio constituye el $80 \%$ del mercurio total en cabello ${ }^{23}$, este consumo que es bastante elevado en esta población, estaría relacionado con las concentraciones encontradas en los peces y aunque estas concentraciones no superaron los limite admisibles, se sabe que la exposición crónica, así sea a niveles bajos se refleja en concentraciones de mercurio elevados en cabello. Las concentraciones de mercurio encontradas tanto en la población como los observados en los peces muestreados, están directamente relacionados con la cercanía que existe entre la población y los distritos mineros de la región, como describieron Interdonato, et $a^{4}{ }^{48}$, en un estudio llevado a cabo en una región de Italia con adolescentes entre 12 y 14 años, en el cual se encontró que las concentraciones de metales pesados en esta población estaban relacionados con la cercanía a distritos industriales que vertían residuos de metales como plomo, mercurio, cadmio, entre otros.

Los síntomas más frecuentemente relacionados con la intoxicación por mercurio son entre otros, nerviosismo, insomnio, pérdida de memoria, debilidad, caída de cabello $^{41}$. La mayoría de los estudios realizados en población infantil expuesta a mercurio referencian daños a nivel neurológico, por lo que la calidad de vida se ve afectada en esta población por la persistencia de la exposición prenatal a mercurio. En nuestro estudio este componente neurológico no se estudió, lo que hace necesario que se realicen otros estudios en esta población, en los que se incluya la evaluación neurológica, para comparar con otros estudios realizados en poblaciones que viven cerca a zonas de explotación aurífera, en los que se han estudiado efectos a nivel neurológico por la exposición a mercurio.

El insomnio y el temblor fueron los dos síntomas más comúnmente expresados por los participantes, lo que coincide con el estudio que hicieron O'Reilly, et al., en población de niños y adolescentes en Zimbawe, donde analizaron muestras de sangre y cabello y encontraron que estos niños presentaron ataxia con temblor, entre otros síntomas ${ }^{4}$. Así mismo, insomnio, dolor de estómago, diarrea fueron síntomas típicos en los encuestados que viven cerca de la zona de extracción de oro, en un estudio realizado por Mega, et al., quienes concluyeron que la minería artesanal tiene un gran impacto en las concentración de mercurio en el cuerpo humano ${ }^{1}$.

\section{Conclusiones}

La población de nuestro estudio ha vivido desde siempre en áreas cercanas a la extracción minera y por supuesto se contaminan ambientalmente, teniendo en cuenta que sus padres laboran en la minería o sus residencias están ubicadas cerca de compraventas y respiran los vapores de mercurio producidos al momento de la quema de amalgama. Así, el tiempo de residencia en los lugares donde se realiza extracción de oro resulta ser un factor de riesgo de exposición a mercurio. 
Infortunadamente, son pocas o no existen las medidas y controles que se requieren para evitar la contaminación de compartimentos ambientales como suelo, aire y agua con mercurio y, en consecuencia, altas concentraciones de este metal persisten y se integran en la cadena alimentaria de los peces y a los consumidores de los mismos.

La frecuencia en los síntomas relacionados con la intoxicación por mercurio resultó elevada en ambos grupos, ratificando que las concentraciones de mercurio en esta población están produciendo signos y síntomas relacionados con intoxicación

Se espera que el presente trabajo contribuya a la identificación de las condiciones que determinan las concentraciones de mercurio en población expuesta, para que se optimicen las condiciones en las que se desarrolla la actividad minera y buscar alternativas más seguras, en el ejercicio de esta labor.

\section{Agradecimientos}

Expresamos nuestros agradecimientos a las personas que participaron en el estudio, así como a los profesionales de las Direcciones Vigilancia y Análisis de Riesgo en Salud Pública e Investigación en Salud Pública del Instituto Nacional de Salud, a los profesionales de las Secretarias de Salud Departamentales y Municipales, por sus valiosos aportes en la obtención de la información en trabajo de campo. A las Direcciones de Vigilancia y Análisis de Riesgo en Salud Pública e Investigación en Salud Pública; Grupos de Factores de Riesgo Ambiental y Salud Ocupacional y Ambiental del Instituto Nacional de Salud por la financiación.

\section{Consideraciones éticas}

Esta investigación tuvo en cuenta las consideraciones éticas planteadas en la Resolución 8430 de 1993 del Ministerio de Salud de Colombia. A los acudientes de los menores se les informó sobre el estudio, los objetivos y beneficios de la investigación y una vez aceptaron participar, se les solicitó la firma del asentimiento informado y con los menores se procedió a la firmar del mismo. Este estudio fue aprobado por el Comité técnico y de ética de la Universidad de los Andes, Colombia (Número de aprobación 459/2015).

\section{Conflicto de interes}

Las autoras no tienen conflictos de intereses que declarar.

\section{Referencias}

1. Sari M, Inoue T, Matsumoto Y, Yokota K. Measuring total mercury due to small-scale gold mining activities to determine community vulnerability in Cihonje, Central Java, Indonesia. Water Sci Technol. 2016; 73(2): 437-444. doi: 10.2166/wst.2015.503.

2. Basri, Sakakibara M, Sera K. Current mercury exposure from artisanal and small-scale gold mining in Bombana, Southeast Sulawesi, Indonesia-Future Significant Health Risks. Toxics. 2017; 5(7): 1-10. doi: https://doi.org/10.3390/toxics5010007.

3. Defensoría de Pueblo. La Minería de Hecho en Colombia. Bogotá, Colombia; 2010. (Defensoría Delegada para los Derechos Colectivos y del Ambiente).

4. Prieto J, González C, Román A, Prieto F. Contaminación y fitotoxicidad en plantas por metales pesados provenientes de suelos y agua. Trop Subtrop Agroecosystems. 2009;10(1): 29-44.

5. Gibb H, O'Leary KG. Mercury exposure and health impacts among individuals in the artisanal and smallscale gold mining community: a comprehensive review. Env Health Perspect. 2014; 122: 667-672. doi: 10.1289/ehp.1307864.

6. PNUMA. Informe relativo a la información sobre sistemas armonizados para medir la carga de mercurio. Chiba (Japón); 2010. Report No.: UNEP(DTIE)/Hg/INC. $2 / 6$.

7. Gilbert S. A small dose of toxicology. The health effects of common chemicals. CRC Press LLC. Vol. A small dose of mercury or An introduction to the health effects of mercury. 2004. 259 p.

8. Marinho JS, Lima MO, de Oliveira Santos EC, de Jesus IM, da Conceição N Pinheiro M, Alves $\mathrm{CN}$, et al. Mercury Speciation in hair of children in three communities of the Amazon, Brazil. Biomed Res Int. 2014; (945963). doi: http://dx.doi. org/10.1155/2014/945963.

9. Molina CF, Arango C, Serna M. Mercurio: implicaciones en la salud y el medio ambiente. Retel. 2010; 7-19.

10. Khatoon-Abadi A, Sheikh Hoseini A, Khalili B. Effect of mercury on the human healthand environment: an overview. Int J Food Saf Nutr Public Health. 2008; 1(1): 33-50. doi: https://doi. org/10.1504/IJFSNPH.2008.018854.

11. Olivero-Verbel J, Duarte D, Echenique M, Guette J, Johnson-Restrepo B, Parsons PJ. Blood lead levels in children aged 5-9 years living in Cartagena, Colombia. Sci Total Environ. 2007; 372(2-3): 707716. doi: 10.1016/j.scitotenv.2006.10.025.

12. Poulin J, Gibb H. Mercurio: Evaluación de la carga 
de morbilidad ambiental a nivel nacional y local. Organización Mundial de la Salud. Ginebra: PrüssÜstün A; 2008. (Carga de Morbilidad Ambiental).

13. Banco de la Republica. La Mojana: riqueza natural y potencial económico. Documento de Trabajo sobre economía regional. Colombia; 2004.

14. Du B, Li P, Feng X, Qiu G, Zhou J, Maurice L. Mercury exposure in children of the Wanshan mercury mining area, Guizhou, China. Int J Environ Res Public Health. 2016; 13(11). doi: 10.3390/ ijerph13111107.

15. Choi J, Chang J, Hong J, Shin S, Park J, Oh S. LowLevel toxic metal exposure in healthy weaning-age infants: association with growth, dietary intake, and iron deficiency. Int J Env Res Public Health. 2017; 14(4): 388. doi: 10.3390/ijerph14040388.

16. Gustin K, Tofail F, Mehrin F, Levi M, Vahter M, Kippler M. Methylmercury exposure and cognitive abilities and behavior at 10 years of age. Environ Int. 2017; 102: 97-105. doi: 10.1016/j. envint.2017.02.004.

17. ATSDR. Reseña Toxicológica del Mercurio. Agencia para Sustancias Tóxicas y el Registro de Enfermedades. 1999.

18. Rogers HS, McCullough J, Kieszak S, Caldwell KL, Jones RL. Exposure assessment of young children living in Chicago communities with historic reports of ritualistic use of mercury. Clin Toxicol. 2007; 45(3): 240-247. doi: 10.1080/15563650601031643.

19. Hudson PJ, Vogt RL, Brondum J, Witherell L, Myers G. Elemental mercury exposure among children of thermometer plant workers. Pediatrics. 1987; 79(6): 935-938.

20. Zheng L, Sanders A, Saland J, Wrighta R, Arora M. Environmental exposures and pediatric kidney function and disease: a systematic review. Environ Res. 2017; 158: 625-648. doi: 10.1016/j. envres.2017.06.029.

21. Grandjean P, Weihe P, Debes F, Choi AL, BudtzJørgensen E. Neurotoxicity from prenatal and postnatal exposure to methylmercury. Neurotoxicol Teratol. 2014; 43: 39-44. doi: 10.1016/j. ntt.2014.03.004.

22. Ozuah P. Folk use of elemental mercury: a potential hazard for children? J Natl Med Assoc. 2001; 93(9): 320-323.

23. Ruggieri F, Majorani C, Domanico F, Alimonti A. Mercury in children: current state on exposure through human biomonitoring studies. Int J Env Res Public Health. 2017; 14(5): 519. doi: 10.3390/ ijerph14050519.

24. Vargas F. La contaminación ambiental como factor determinante de la salud. Rev Esp Salud Publica.
2005; 79(2): 117-127.

25. OMS. Las consecuencias de la contaminación ambiental: 1,7 millones de defunciones infantiles anuales, según la OMS; 2017.

26. Counter S, Buchanan L, Ortega F. Acoustic stapedius muscle reflex in mercury-exposed Andean children and adults. Acta Otolaryngol. 2012; 132(1): 51-63. doi: 10.3109/00016489.2011.617778.

27. Health Canada Mercury Issues Task Group. Mercury Your health and the environment a resource tool. Canada; 2004 p. 54.

28. OMS, IPCS. Elemental mercury and inorganic mercury compounds: human health aspects. Geneve; 2003.

29. Escobar M, Zabala M. Perfil neuropsicológico de escolares con trastornos específicos del aprendizaje de instituciones educativas de Barranquilla, Colombia. Acta Neurol Colomb. 2008; 24: 63-73.

30. Rosselli M,Ardila A. Predictores neuropsicológicos de la lectura en español. Rev Neurol. 2006; 42: 202-210.

31. United Nations Environment Programme. Towards a pollution-free planet. $3 \mathrm{rd}$ session of the United Nations Environment Assembly. Nairobi, Kenya; 2017.

32. Andreoli V, Sprovieri F. Genetic Aspects of Susceptibility to Mercury Toxicity: an Overview. Int J Env Res Public Health. 2017; 14(1): 14-93. doi: 10.3390/ijerph 14010093.

33. Pesh P, Wilhelm M, Rostek U, Schmitz N, Weishoff $\mathrm{M}$, Ranft U, et al. Mercury concentration in urine, scalp hair and saliva in children from Germany. J Expo Anal Environ Epydemiol. 2002; 12(4): 252258. doi:10.1038/sj.jea.7500228.

34. Mc Dowell M, Dillon Ch, Osterloh J, Bolgr M, Pellzzari E, Fernando R, et al. Hair mercury levels in U.S. children and women of childbearing Age: reference range data from NHANES 2999-2000. Environ Health Perspect. 2004; 112(111): 11651171. doi: 10.1289/ehp.7046.

35. Kim SA, Jeon CK, Paek DM. Hair mercury concentrations of children and mothers in Korea: Implication for exposure and evaluation. Sci Total Environ. 2008; 402(1): 36-42. doi: 10.1016/j. scitotenv.2008.04.010.

36. Kusanagi E, Takamura H, Chen SJ, Adachi M, Hoshi N. Children's Hair Mercury concentrations and seafood consumption in five regions of Japan. Arch Env Contam Toxicol. 2018; 74(2): 259-272. doi: 10.1007/s00244-017-0502-x.

37. Ou L, Chen C, Chen L, Wang H, Yang T, Xie H, et al. Lowlevel prenatal mercury exposure in north china: an exploratory study of anthropometric 
effects. Env Sci Technol. 2015; 49(11): 6899-6908. doi: 10.1021/es5055868.

38. Castoldi AF, Coccini T, Ceccatelli S. Neurotoxicity and molecular effects of methylmercury. Brain Res Bull. 2001; 55(2): 197-203. doi: https://doi. org/10.1016/S0361-9230 (01)00458-0.

39. Ekino S, Susa M, Ninomiya T, Imamura K, Kitamura T. Minamata disease revisited: An update on the acute and chronic manifestations of methyl mercury poisoning. J Neurol Sci. 2007; 262(1-2): 131-44. doi: 10.1016/j.jns.2007.06.036.

40. UNEP. Global Mercury Assessment. Switzerland: United Nations Environment Programme; 2013. (Sources, Emissions, Releases and Environmental Transport).

41. Calao C, Marrugo JL. Efectos genotóxicos asociados a metales pesados en una población humana de la región de La Mojana, Colombia, 2013. Biomédica. 2015; 35(Supl 2): 139-151. doi: http://dx.doi. org/10.7705/biomedica.v35i0.2392.

42. Vargas B, ML, Quiroz P, CM. Alteraciones neuropsicológicas en escolares de un municipio con niveles elevados de vapor de mercurio medioambiental, Colombia, 2008-2009. Rev Fac Nac Salud Pública. 2011; 29(4): 461-468.

43. WHO, UNEP, DTIE CHEMICALS BRANCH. Guidance for identifying populations at risk from mercury exposure. Geneva, Switzerland; 2008.

44. Gracia L, Marrugo JL, Alvis E. Mercury contamination in humans and fishes in the municipality of Ayapel, Córdoba, Colombia, 2009. Rev Fac Nac Salud Pública. 2010; 28: 118-124. doi: 10.17533/udea.rfnsp.

45. Bose-O'Reilly S, Lettmeier B, Matteucci M, Gothe R, Beinhoff C, Siebert U, et al. Mercury as a serious health hazard for children in gold mining areas. Environ Res. 2008; 107(1): 89-97. doi: 10.1289/ ehp.1307864.

46. OMS. Mercurio: evaluando la carga de la enfermedad a nivel nacional y local. Serie sobre carga ambiental de enfermedades. Ginebra; 2008. Report No. 16.

47. Abdul Samad N, Md Isa Z, Hod R. Mercury hair concentration among primary school children in Malaysia. Child Basel. 2017; 4(12). doi: 10.3390/ children4120109.

48. Interdonato M, Bitto A, Pizzino G, Irrera N, Pallio G, Mecchio A, et al. Levels of Heavy Metals in Adolescents Living in the Industrialised Area of Milazzo-Valle del Mela (Northern Sicily). J Environ Public Health. 2014; 1:1-9. doi: 10.1155/2014/326845.

\section{ABREVIATURAS}

Hg: mercurio

OMS: Organización Mundial de la Salud

\section{UNIDADES DE MEDIDA}

$\mathrm{mg} / \mathrm{kg}$ : miligramo por kilogramo

$\mathrm{mg} / \mathrm{g}$ : miligramo por gramo

$\mathrm{mg} / \mathrm{L}$ : miligramo por litro 